\title{
Drying Mediated Aggregation Pattern of CdTe QDs towards the Fabrication of Molecular Devices
}

\author{
A. Arivarasan, S. Bharathi, S. Arunpandiyan, S. Ezhil Arasi, P. Devendran
}

\begin{abstract}
Drying mediated self-aggregation patterns of CdTe colloidal QDs through simple evaporation method was reported. Thioglycolic acid (TGA) capped CdTe QDs were prepared in aqueous phase through colloidal route and their structural, optical and morphological behaviors were studied. The drying patterns of CdTe QDs were fabricated under a constant evaporation rate. The coffee ring effect and the drying pattern formations were studied for the TGA capped CdTe colloidal QDs suspension in an aqueous medium. The structural behavior of the CdTe QDs and the drying patterns were revealed by the $X$-ray diffraction analysis. The size-tunable optical properties of the prepared CdTe QDs were studied by the optical absorption and emission spectroscopy. The capping effects of TGA on CdTe QDs were confirmed by the FT-IR analysis. The surface morphology of the TGA capped CdTe QDs and the drying mediated aggregation patterns of CdTe colloidal suspension were studied by the scanning electron microscopy analysis. The drying mediated patterns from the evaporation technique were suggested as a simple technique for the fabrication of molecular devices.
\end{abstract}

Keywords: Colloidal quantum dots; Drying patterns; Self-aggregation; Evaporation technique; SEM.

\section{INTRODUCTION}

$\mathrm{Q}$ uantum dots attract large attention for its size tuneable optical properties due to their quantum confinement effects [1]. Epitaxial patterned QDs were significantly used in device fabrication; however, their device applications are limited due to the high cost of production, complicated lithography procedures and also their limitations in mass production. Colloidal QDs may overcome these difficulties and they can be modified into any form by controlling the reaction parameters [2]. Cadmium Telluride (CdTe) QDs was one of the widely studied II-VI semiconducting nanostructures for its optical, electronic and biological applications. CdTe QDs

Revised Manuscript Received on December 18, 2019.

* Correspondence Author

A. Arivarasan*, Department of Physics, Kalasalingam Academy of Research and Higher Education, Krishnankoil, Tamil Nadu 626126, India. Email: arivarasan.nanotech@gmail.com

S. Bharathi, NGSeq Analytics LLC, San Diego, California, United States of America. Email: shalu.vaishu@gmail.com

S. Arunpandiyan, Department of Physics, Kalasalingam Academy of Research and Higher Education, Krishnankoil, Tamil Nadu 626126, India. Email: arunpandiyan5413@gmail.com

S. Ezhil Arasi, Department of Physics, Kalasalingam Academy of Research and Higher Education, Krishnankoil, Tamil Nadu 626126, India. Email: arasi1105@gmail.com

P. Devendran, Department of Physics, Kalasalingam Academy of Research and Higher Education, Krishnankoil, Tamil Nadu 626126, India. Email: pdevavenmani@gmail.com have a more intensive attraction than that of CdTe nanowires and nanorods due to their higher surface to volume ratio. CdTe QDs was optically active over the visible region depending upon the size of the QDs [3]. Due to its intermediate band gap $(\mathrm{Eg}=1.45 \mathrm{eV}$ for bulk $\mathrm{CdTe})$ and higher fluorescent behavior, CdTe QDs were utilized in optoelectronic devices, biological imaging, and spintronic applications. Even though the colloidal QDs have numerous advantages over the epitaxial QDs, the patterning of colloidal quantum dots is still remains as a challenging task. The lack of patterning design results in the reduction of the possible application of colloidal QDs in device fabrications.

Recently the coffee ring effect in drying a colloidal suspension made a considerable interest in the formation of drying patterns $[4,5,6]$. In this effect, the concentration of the particles was increased and pulled towards the edges by the continuous evaporation of the solvents $[7,8,9]$. The Marangoni flow, a circulatory motion of reversed particles towards the center of the droplets was also observed during the evaporation of the droplets. It reduces the coffee ring effect and results in the formation of drying mediated patterns in the center [10]. Evaporation temperature, contact angle and the surface behavior of the substrate weare the major factors which control the properties of the drying patterns. By controlling these reaction conditions, the size and shape of the aggregation pattern can be modified [11, 12, 13]. Deep understanding about the pattern formation was necessary to design the aggregation patterns for potential application Recently the aggregation patterns of gold nanoparticles [14], carbon Nanotubes [15-18], colloidal silica [19] and also the aggregation patterns of polystyrene micro and nano spheres in aqueous phase were studied. Similar to that the aggregation patterns of some organic molecules like DNA [20], lysozyme and serum albumin $[18,21,22]$ were also studied. Evaporative lithography is a promising tool to create the specified aggregation patterns for potential applications [23, 24]. The complex aggregation patterns of colloidal quantum dots were the most promising candidate for molecular devices.

In this work, the complex aggregation pattern of thiol capped CdTe QDs was prepared by controlling the evaporation temperature. The size dependent drying mediated complex aggregation pattern of $\mathrm{CdTe}$ QDs on the hydrophobic surface was prepared and the effect of particle size on the surface behavior of the drying pattern at a constant evaporation rate was studied. 
The mechanism of pattern formation depends upon the evaporation temperature was clearly explained. The main objective of the work was to design the different aggregation patterns of CdTe QDs for suitable molecular devices.

\section{MATERIALS AND METHODS}

\section{A. Materials Used}

Cadmium chloride monohydrate $\left(\mathrm{CdCl}_{2} \cdot \mathrm{H}_{2} \mathrm{O}, 99.9 \%\right)$, Tri sodium citrate and sodium borohydride $\left(\mathrm{NaBH}_{4}, 99.9 \%\right)$ were purchased from Merck Chemicals, India. Potassium tellurite $\left(\mathrm{K}_{2} \mathrm{TeO}_{3}\right)$ and thio glycolic acid (TGA) were purchased from Alfa Aesar chemicals. All the other standard chemicals were purchased from $\mathrm{CDH}$ chemicals, India. Millipore water with the resistivity of $18 \mathrm{M} \Omega$ was used as the solvent for the synthesis procedure.

\section{B. Synthesis procedure}

TGA capped CdTe QDs was prepared in aqueous phase by using potassium tellurite and cadmium chloride as the precursors $[25,26]$. The molarity ratio of the precursors and the capping agent $\mathrm{Cd}^{2+}$ : TGA: $\mathrm{Te}^{2-}$ was 1:2.5:0.5. The $\mathrm{pH}$ of the solution was maintained at 9 by the drop wise addition of $2 \mathrm{M} \mathrm{NaOH}$ solution. A clear color change was observed from transparent to light green on addition of potassium tellurite and sodium borohydride in aqueous $\mathrm{CdCl}_{2}$ solution, which indicates the formation of $\mathrm{CdTe}$ monomers. The resultant solution with light green color was refluxed in a round bottomed flask at $100^{\circ} \mathrm{C}$ with a condenser attached. The change in color between light green and dark red indicates the size variation of the CdTe QDs depending on the refluxing time. The aliquots were collected at different refluxing times for optical studies. The prepared QDs was precipitated and washed with acetone and water repeatedly. The purified CdTe QDs were used for structural and surface analysis.

The drying patterns are created on the glass substrate by the evaporation technique. The glass substrate with the dimension of $2.5 \mathrm{~cm} \times 2.5 \mathrm{~cm}$ was thoroughly washed with acetone and Millipore water and heated at $90^{\circ} \mathrm{C}$ for $20 \mathrm{~min}$ to remove the water and organic residuals. The prepared QDs were re-dissolved in water and the resultant solution was sonicated for $30 \mathrm{~min} .10 \mu \mathrm{L}$ of the prepared colloidal QDs solution was deposited over the glass substrate at room temperature. The deposited CdTe QDs were dried at $90^{\circ} \mathrm{C}$ to study the evaporation temperature dependent surface behavior of the resultant pattern.

\section{RESULTS AND DISCUSSION}

\section{A. X-Ray diffraction (XRD) analysis}

The XRD patterns of TGA capped CdTe QDs were shown in figure 1 (a). TGA capped CdTe QDs have the diffraction peaks at the angles of $25.42^{\circ}, 30.8^{\circ}, 43.8^{\circ}$ and $51.7^{\circ}$ corresponding to the crystalline planes of (111), (200), (220) and (311) respectively. This was in good agreement with the cubic zinc blende crystalline structure of bulk CdTe. The broad diffraction peaks reveal the nano dimension of the

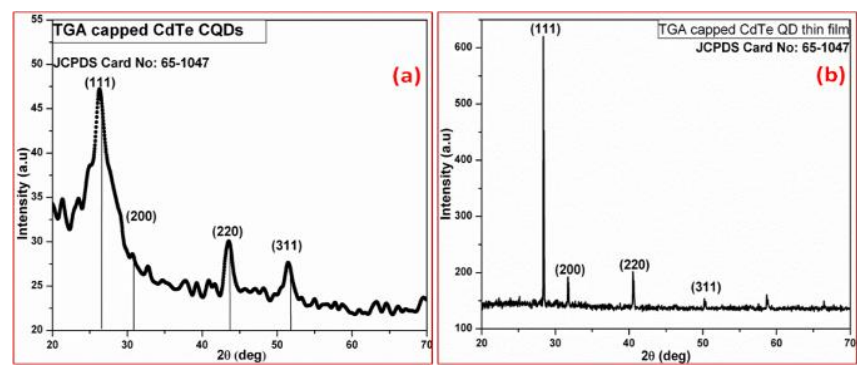

Fig. 1.XRD pattern of (a) TGA capped CdTe QDs and (b) complex aggregation pattern of CdTe QDs.

prepared CdTe QDs and the average crystalline size calculated from Scherrer's formula was $3 \mathrm{~nm}$. The similar diffraction pattern was observed for the aggregation patterns of the CdTe QDs, except a small shift in peak position due to the strain induced defects (figure 1b). The full width at half maximum (FWHM) values of the diffraction peaks was greatly reduced in the aggregation patterns. This clearly indicates that the crystalline size of the aggregation patterns was very high when compared with the CdTe QDs due to the re-crystallization of the QDs.

\section{B. Optical Studies}

The UV-vis absorption spectra and their corresponding fluorescence emission spectra of the prepared QDs in different refluxing times from $30 \mathrm{~min}$ to $9 \mathrm{~h}$ were shown in figure 2. The existence of well resolved absorption maxima ranges between $410 \mathrm{~nm}$ and $470 \mathrm{~nm}$ were observed in the absorption spectra (figure 2a). A strong red shift was observed on the absorption band edge with the increase in refluxing time, which indicates the band gap of the prepared QDs were decreased with the increasing in refluxing time. The change in the band gap indicates that the size of the QDs was increased with the refluxing time. The band gap of the prepared QDs lies between $2.5 \mathrm{eV}$ and $2.8 \mathrm{eV}$. Figure 2(b) represents the normalized fluorescence spectra of the prepared CdTe QDs. All the prepared samples showed well pronounced emission maxima with a strong red shift on the increment of refluxing time. A strong emission peak with lower FWHM values was observed at the refluxing time of $5 \mathrm{~h}$ indicates the homogeneous size distribution of the QDs. The higher FWHM value indicates the inhomogeneous size distributions of CdTe QDs. The quantum yield calculation shows the highest quantum yield of $64 \%$ at the refluxing time of $5 \mathrm{~h}$ and the longer refluxing time results in the decrement of QE.

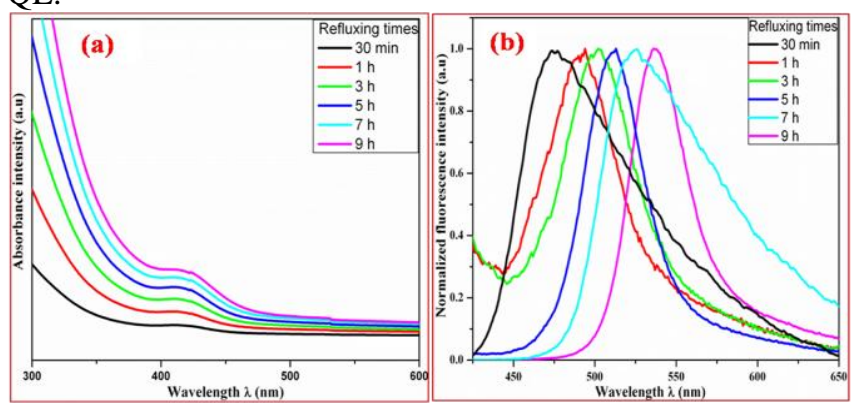

Fig. 2.(a) Normalized fluorescence spectra and (b) UV-Vis absorption spectra of TGA capped CdTe QDs. 


\section{FT-IR analysis}

The FT-IR spectra and the fluorescence images of TGA capped CdTe QDs were shown in figure3. The fluorescence images (Figure 3(a)) of the prepared CdTe QDs for different refluxing times under UV light illumination was an evidence for size dependent optical properties of the CdTe QDs. The fluorescence emission color of the CdTe QDs was varied in between violet and dark red depending upon the duration of the refluxing time. It clearly indicates that the size of the CdTe QDs was increased with the refluxing time. FT-IR spectra of TGA capped CdTe QDs and the aggregation patterns (Figure 3 (b)) clearly demonstrate the existence of the thiol capping agent on the prepared QDs. The broad absorption peaks at around $3450 \mathrm{~cm}^{-1}$ was assigned to the $\mathrm{OH}$ vibration of the presence of water molecules. The $\mathrm{CH}_{2}$ vibrations $\left(2940 \mathrm{~cm}^{-1}\right)$ of TGA was shifted towards the lower frequency $\left(2925 \mathrm{~cm}^{-1}\right)$ in TGA capped CdTe QDs and the aggregation patterns. This may be attributed to the effect of CdTe solid surface on the absorbed state of TGA. The S-H vibrations of pure TGA at around $2500 \mathrm{~cm}^{-1}$ and $2600 \mathrm{~cm}^{-1}$ were completely disappeared in TGA capped CdTe QDs and in the aggregation patterns [27]. The presence of two sharp absorption peaks at around $1653 \mathrm{~cm}^{-1}$ and $1358 \mathrm{~cm}^{-1}$ due to the symmetric and asymmetric vibrations of $\mathrm{COO}$ - clearly indicates that the thiol capping agent were chemisorped on the surface of CdTe QDs. The absorption peak at around $711 \mathrm{~cm}^{-1}$ were assigned to the CdTe QDs formation by Cd-SR bonds.
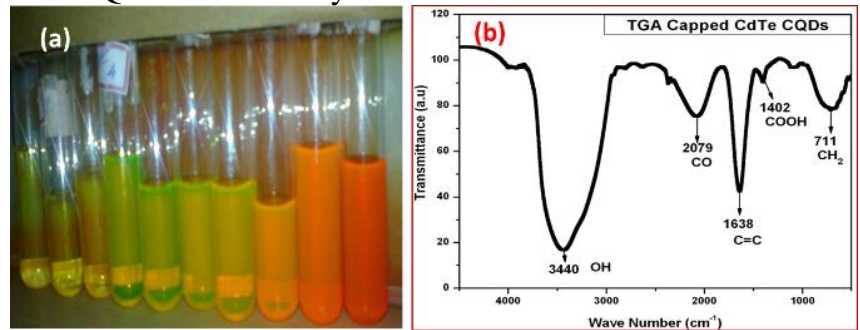

Fig. 3.(a) Size dependent fluorescence images of CdTe QDs under UV light illumination and (b) FT-IR spectrum of TGA capped CdTe QDs and the aggregation patterns.

\section{Surface analysis-Optical microscopy and SEM}

The coffee ring effect and the formation of self-aggregation patterns due to the evaporation of prepared CdTe colloidal QDs solution were studied by the optical microscopy analysis. Figure 4 depicts the optical microscopy images of the prepared CdTe QDs at controlled evaporation. It shows the formation of coffe rings at the edges of the droplets. The stability of the particles was decided by the smaller particles whereas the larger particles move towards the peripherals of the drop by the shear forces. The smaller thermal agitation and the higher cohesion of the larger particles are responsible for the outward movement. The outward ripening of the particles was responsible for this coffee ring effect [4]. Thus, the inter-particles attractive van der Waals forces were responsible for these increments of cohesive energy of the aggregation patterns. At the same time, the smaller particles are assembled together to form the aggregation patterns at the centre of the droplets, which is clearly observed from the optical images. The schematic representation to explain the formation of coffee ring effect by controlled evopation techniques were shown in figure 5 .

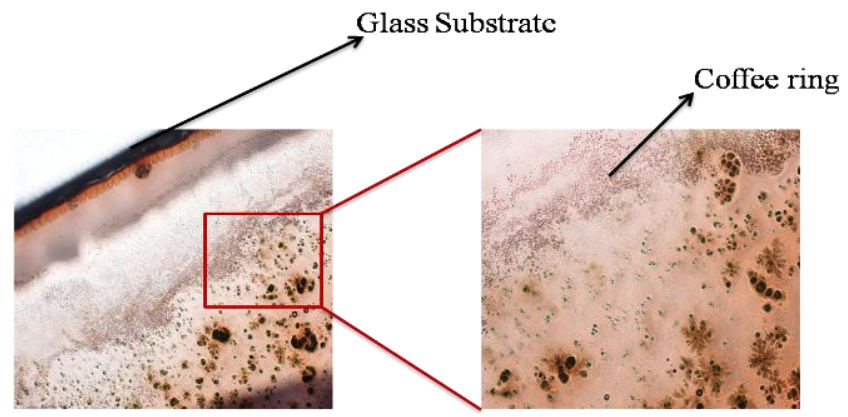

Fig. 4.Cyclic voltammogram of the $\mathrm{Fe}_{2} \mathrm{O}_{3} / \mathrm{CeO}_{2}$ nanocomposites.

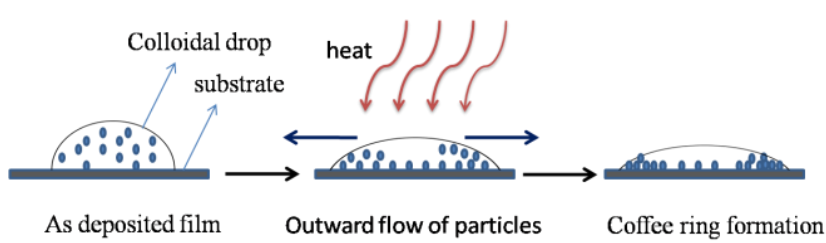

Fig. 5.Schematic representation of coffee ring effect and self aggregation patterns

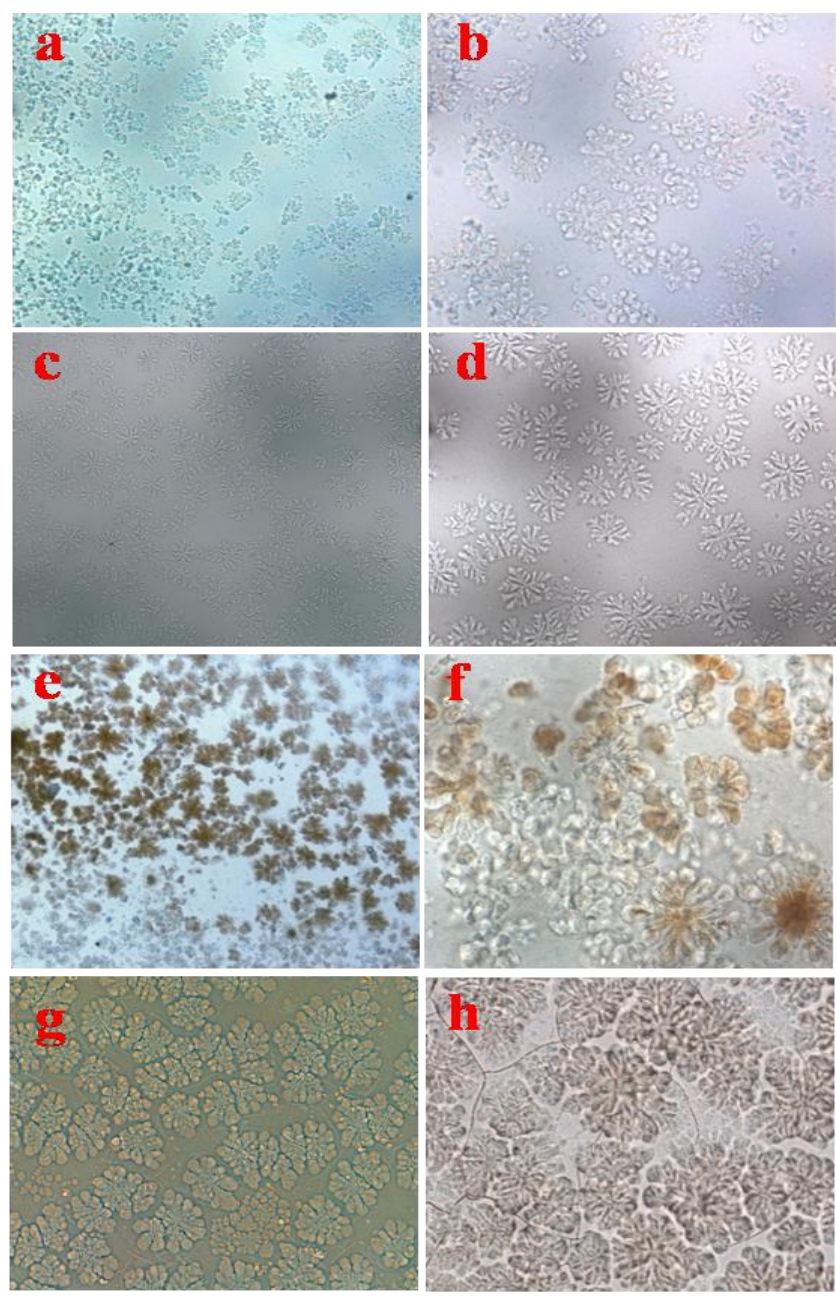

Fig. 6.Optical images of drying patterns for various sized CdTe QDs. 
The optical images of drying mediated aggregation patterns of CdTe QDs with different sizes were shown in Figure 6. The sizes of the QDs are (a\&b) $2 \mathrm{~nm},(\mathrm{c} \& \mathrm{~d}), 4 \mathrm{~nm}(\mathrm{e} \& \mathrm{f}), 6 \mathrm{~nm}$ and $(\mathrm{g} \& \mathrm{~h}) 8 \mathrm{~nm}$ respectively. Different aggregation patterns were observed in the optical images with corresponding to the size of the QDs, which clearly indicates that the aggregation patterns are size dependent. From the optical images, it is concluded that the colloidal solution with smaller sized quantum dots (2 to $4 \mathrm{~nm}$ ) produce the well-ordered aggregation patterns, when compared to larger sized QDs (6 to $8 \mathrm{~nm}$ ).

Fig.7 The surface morphology of the prepared drying mediated aggregation patterns was studied by SEM analysis. The images (a) and (b) in figure 7, represent the SEM images with two different magnifications of TGA capped CdTe QDs in powder form at room temperature. The SEM images clearly show the presence of spherical like colloidal QDs and it looks like clusters due to small agglomeration. According these images, the prepared CdTe QDs have well defined boundaries with a spherical shape. There are no defined patterns were observed in powder form.

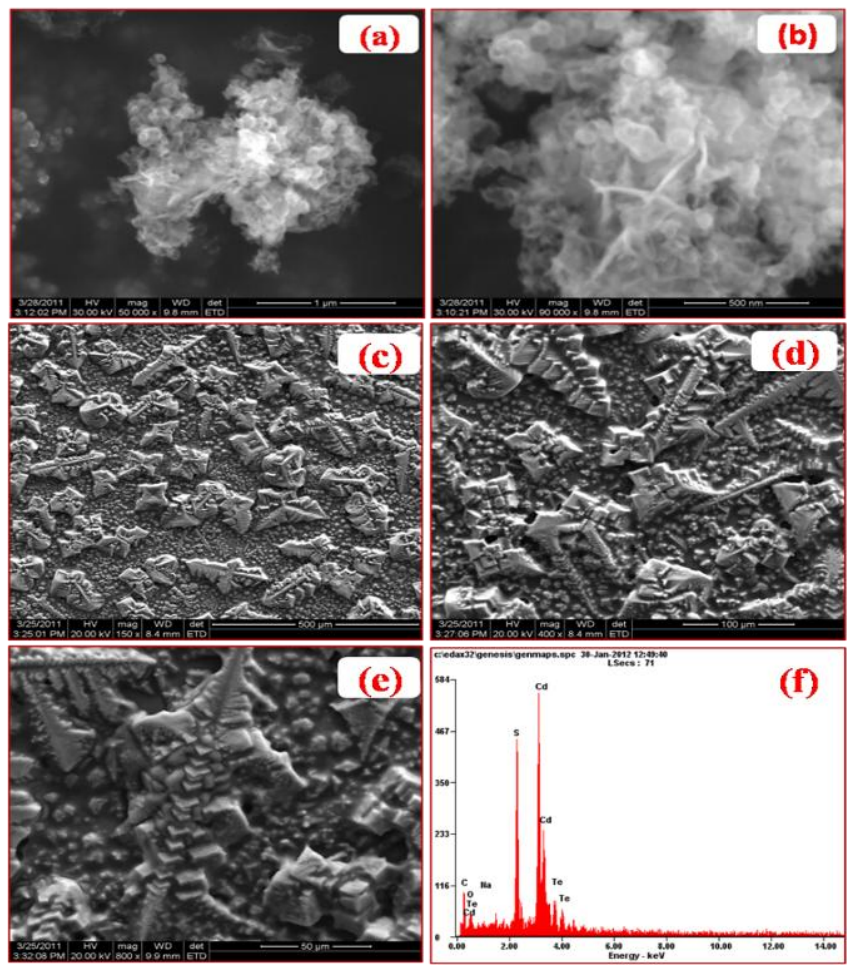

Fig. 7.SEM images of (a\&b) powdered CdTe QDs (c,d\&e) aggregation patterns in different magnifications and (f) EDAX pattern of CdTe QDs.

The images (c), (d) and (e) in figure 7 represents the SEM images of complex aggregation patterns due the controlled evaporation of CdTe QDs under different magnifications. The SEM images reveals that the dendrite like aggregation patterns was formed during the drying process of $3 \mathrm{~nm}$ sized CdTe QDs by the controlled solvent evaporation technique. The colloidal crystallization is the predominant process occurred by the evaporation process results in the formation of aggregation patterns. Increase in thermal energy results in the disordered orientation of QDs to the aggregation patterns. At higher evaporation rate the smaller QDs aggregates into patterns rather than bigger QDs. The complex nature of the aggregation pattern was gradually decreased with increasing the size of the QDs [13]. Figure 7 (d) represents the EDAX spectrum of TGA capped CdTe QDs. The EDAX spectrum clearly indicates the presence of $\mathrm{Cd}$ and Te compounds along with the sulfur functional group of the thiol capping agent in TGA capped CdTe QDs.

The schematic representation of evaporative lithographic process to produce the complex aggregation patterns were shown in figure 8 . In this process evaporation temperature and the distance between the mask and the film were the crucial factors to decide the shape of the aggregation patterns $[23,24]$. The size and the area of the aggregation patterns were controlled by the area of the hole in the mask. By controlling the evaporation parameters, the desired patterns were created on the surface for molecular devices.

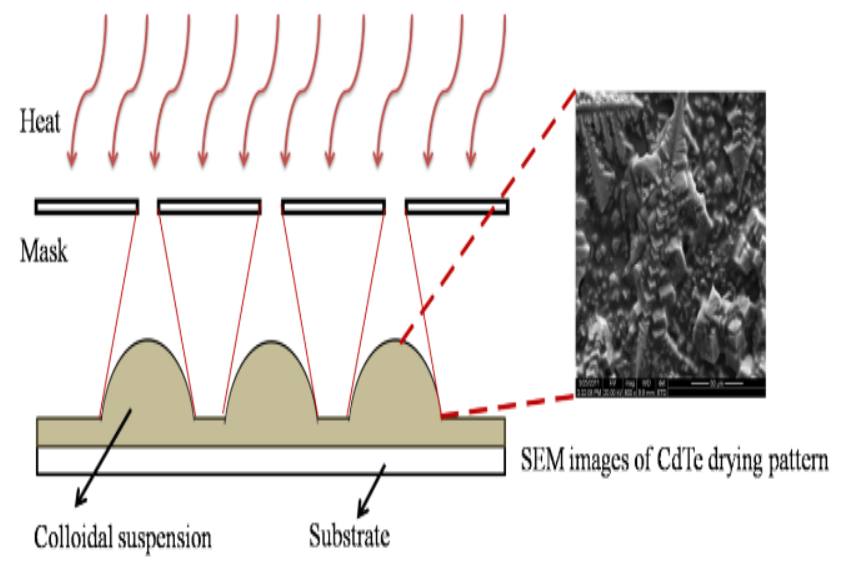

Fig. 8.Fabrication of aggregation patterns

\section{CONCLUSION}

A simple approach was proposed to produce the drying mediated aggregation patterns of the QDs systems. TGA capped CdTe QDs was produced in colloidal route with the controlled particle size of $3 \mathrm{~nm}$. Drying mediated aggregation patterns of CdTe QDs was produced by the controlled evaporation of the solvent from the colloidal suspension. The structural variations between the pure CdTe QDs and the drying mediated aggregation patterns was studied with the XRD analysis. The average crystalline size of the CdTe QDs (3 nm) was estimated from the Debye's Scherrer's formula. Optical studies clearly indicate the size dependent optical properties of the TGA capped CdTe QDs. Fluorescence images under UV illumination was a clearly evident in the size dependent optical properties of the CdTe QDs. The spherical like quantum dots and the fractal like aggregation patterns of the CdTe QDs was studied from the SEM images. The pattern formation of CdTe QDs through a drying process made a new way to produce the ordered patterns of the QDs and the nanoparticles systems. The ordered patterns may produced by using evaporative lithography techniques. These kinds of ordered patterns of colloidal QDs will play a vital role in the fabrication of next generation molecular devices.

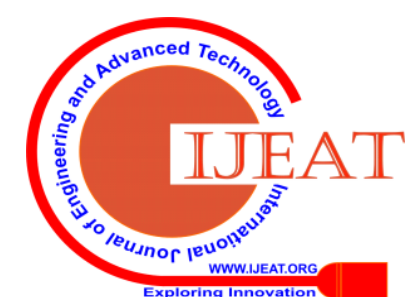




\section{REFERENCES}

1. A. P. Alivisatos, Perspectives on the Physical Chemistry of Semiconductor Nanocrystals, J. Phys. Chem. 1996, 100, 13226-13239

2. Philippe Guyot-Sionnest, Colloidal quantum dots, C. R. Physique, 9 (2008) 777-787

3. Arivarasan A., Sasikala G. and Jayavel R., "In situ synthesis of CdTe:CdS quantum dot nanocomposites for photovoltaics applications", Materials science in semiconductor processing, Vol. 25 (2014), pp. 238-243.

4. Robert D. Deegan, Olgica Bakajin, Todd F. Dupont, Greb Huber, Sidney R. Nagel \& Thomas A. Witten, Capillary flow as the cause of ring stains from dried liquid drops, NATURE ,389,1997, 827-829

5. Robert D. Deegan, Pattern formation in drying drops, Physical Review, 61, 1,2000, 475-485

6. R. D. Deegan, O. Bakajin, T. F. Dupont, G. Huber, S. R. Nagel, and T. A. Witten, "Contact line deposits in an evaporating drop," Physical Review E, vol. 62, no. 1, pp. 756-765, 2000.

7. Samer Darwich, Karine Mougin, and Hamidou Haidara, Complex Aggregation Patterns in Drying Nanocolloidal Suspensions: Size Matters When It Comes to the Thermomechanical Stability of Nanoparticle-Based Structures, Langmuir 2010, 26(22), 16928-16933

8. K. Mougin and H. Haidara, Complex Pattern Formation in Drying Dispersions, Langmuir, 2002, 18, 9566-9569

9. Ziye Xiong, Pattern formation in drying drops of colloidal solutions, Swanson School of Engineering, University of Pittsburgh, 2003

10. H. Hu and R. G. Larson, "Analysis of the Effects of Marangoni Stresses on the Microflow in an Evaporating Sessile Droplet," Langmuir, vol. 21, no. 9, pp. 3972-3980, Apr. 2005.

11. Chris S. Hodges, Yulong Ding, Simon Biggs, The influence of nanoparticles shap on the drying of colloidal suspensions, Journal of Colloid and Interface Science, 352 (2010) 99-106

12. Alexander F Routh, Drying of thin colloidal films, Rep. Prog. Phys., 76 (2013) 046603 (30pp)

13. Samer Darwich Colloidal Gold Nanoparticles: A study of their Drying-mediated Assembly in mesoscale aggregation patterns and of their AFM-assisted Nanomanipulation on model solid surfaces, University of Haute-Alsace, December 14, 2011

14. H. Wei, Z. Wang, J. Zhang, S. House, Y.-G. Gao, L. Yang, H. Robinson, L. H. Tan, H. Xing, C. Hou, I. M. Robertson, J.-M. Zuo, and Y. Lu, "Time-dependent, protein-directed growth of gold nanoparticles within a single crystal of lysozyme", Nature Nanotechnology, vol. 6 , no. 2, pp. 93-97, Jan. 2011.

15. L. Huang, X. Cui, G. Dukovic, and S. P. O Brien, "Self-organizing high-density single-walled carbon nanotube arrays from surfactant suspensions," Nanotechnology, vol. 15, no. 11, pp. 1450-1454, Nov. 2004.

16. H. Ko, S. Peleshanko, and V. V. Tsukruk, "Combing and Bending of Carbon Nanotube Arrays with Confined Microfluidic Flow on Patterned Surfaces," The Journal of Physical Chemistry B, vol. 108, no. 14, pp. 4385-4393, Apr. 2004.

17. R. Duggal, F. Hussain, and M. Pasquali, "Self-Assembly of Single-Walled Carbon Nanotubes into a Sheet by Drop Drying," Advanced Materials, vol. 18, no. 1, pp. 29-34, Jan. 2006.

18. J.-U. Park, M. A. Meitl, S.-H. Hur, M. L. Usrey, M. S. Strano, P. J. A. Kenis, and J. A. Rogers, "In Situ Deposition and Patterning of Single-Walled Carbon Nanotubes by Laminar Flow and Controlled Flocculation in Microfluidic Channels," Angewandte Chemie International Edition, vol. 45, no. 4, pp. 581-585, Jan. 2006.

19. A. A. Vertegel, R. W. Siegel, and J. S. Dordick, "Silica Nanoparticle Size Influences the Structure and Enzymatic Activity of Adsorbed Lysozyme," Langmuir, vol. 20, no. 16, pp. 6800-6807, Aug. 2004.

20. I. I. Smalyukh, O. V. Zribi, J. C. Butler, O. D. Lavrentovich, and G. C. L. Wong, "Structure and Dynamics of Liquid Crystalline Pattern Formation in Drying Droplets of DNA," Phys. Rev. Lett., vol. 96, no. 17, p. 177801, May 2006.

21. D. Brutin, B. Sobac, B. Loquet, and J. Sampol, "Pattern formation in drying drops of blood," Journal of Fluid Mechanics, vol. 667, pp. 85-95, Jan. 2011.

22. C. C. Annarelli, J. Fornazero, J. Bert, and J. Colombani, "Crack patterns in drying protein solution drops," The European Physical Journal E: Soft Matter and Biological Physics, vol. 5, no. 5, pp. 599-603, 2001.

23. Daniel J. Harris, Hua Hu, Jacinta C. Conrad and Jennifer A. Lewis, Patterning Colloidal Films via Evaporative Lithography, Physical Review Letters, 98, 148301-5 (2007)

24. Daniel J. Harris, Jacinta C. Conrad and Jennifer A. Lewis, Evaporative lithographic patterning of binary colloidal films, Phil. Trans. R. Soc. A, (2009) 367, 5157-5165
25. Ayyaswamy Arivarasan, Bharathi Sambandam, V. Vijayaraj, Sasikala Ganapathy, Ramasamy Jayavel, Evaluation of Reaction Parameters Dependent Optical Properties and Its Photovoltaics Performances of CdTe QDs, Journal of Inorganic and Organometallic Polymers and Materials, 28 (2018) 1263-1275.

26. Ayyaswamy Arivarasan, Sasikala Ganapathy, Ali Alsalme, Abdulaziz Alghamdi, Ramasamy Jayavel, "Structural, optical and photovoltaic properties of co-doped CdTe QDs for quantum dots sensitized solar cells", Superlattices and Microstructures, Vol. 88 (2015) pp. 634-638.

27. M.S. Abd El-sadek, S. Moorthy Babu, A controlled approach for synthesizing CdTe@CrOOH (core-shell) composite nanoparticles, Current Applied Physics 11 (2011) 926-932.

\section{AUTHORS PROFILE}

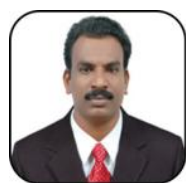

Dr. A. Arivarasan is currently working as Assistant Professor at Kalasalingam academy of Research and education, Virudhunagar, India. He completed his MSc (Physics) degree in Bharathidasan University, Trichy. He received both his M.Tech. (Nanotechnology) and Ph.D. (Nanotechnology) degrees from Anna University in the years of 2010 and 2014, respectively on Quantum Dots for Solar Cell Applications. He has published nearly $20+$ publications in peer reviewed international journals and more than 25 conference proceedings. His current research interests are in the fields of supercapacitor electrodes, redox electrolytes and device fabrications.

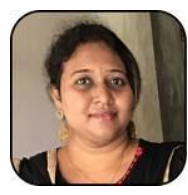

Dr. S. Bharathi is currently working as Managing Director of NGSeq Analytics LLC, San Diego, California, United States of America. She completed her MS (Boitechnology) degree in University of Essex, UK Trichy and received her doctoral degree (Ph.D) from Anna University, Chennai, India in 2015, She has more than five years of teaching and Research experience and she has published nearly $15+$ publications in peer reviewed international journals

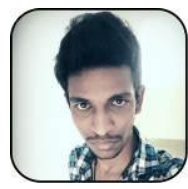

Mr. S. Arunpandiyan received his M.Sc., degree in Physics from Department of Physics, Kalasalingam Academy of Research and Education, Virudhunagar, Tamil Nadu (India) in 2018. Currently, he is a Ph.D. student under the supervision of Dr. A. Arivarasan. His research work focuses on enhancement of supercapacitor performance using redox additive electrolytes. Also, his interests include quantum dot sensitized Solar cells, electrochemical sensors and photoelectrochemical water splitting. He has published four research papers regarding solar cells and supercapacitors in reputed international journals.

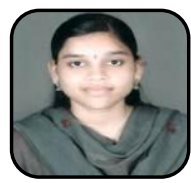

Ms. S. Ezhil Arasi born on 1994 in Erode, Tamilnadu. She obtained her Master degree in Physics in 2017 at Bharathiar University, Coimbatore. She joined Ph.D. in 2017 under the supervision of Dr. A. Arivarasan at Kalasalingam Academy of Research and Education in Virudhunagar district. Her research interest is focused on the area of nanomaterials and carbon-based binary metal nanocomposites synthesis for energy storage device fabrication application

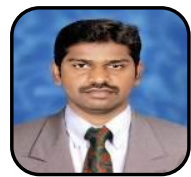

Dr. P. Devendran received his Ph.D degree in Physics from Madras University at Chennai in 2016, developing metal oxides and metal sulfide nano-catalysts for photocatalytic application. After he moved for postdoctoral studies at IRC, Kalasalingam academy of Research and education, he becomes an Assistant Professor of Physics at the same institution and presently began studying metal oxide modified graphene-based materials for energy storage device application and their surface science. He published more than 25+ research articles and book chapters in reputed journals. 